\title{
Overall Planning of Building an Intelligent Automatic System of Cascade Centralized Control Center in River Basins
}

\author{
Gao Qian ${ }^{1,2, a}$ \\ ${ }^{1}$ Department of mechanical engineering, School of mechanical and electrical engineering, Hohai \\ University, Nanjing,Jiangsu, 211100China \\ ${ }^{2}$ School of mechanical and electrical engineering, Jinling Institute of Technology, Nanjing,Jiangsu, \\ 211100China \\ a427999947@qq.com
}

Keywords: Overall Planning of Building an Intelligent Automatic System of Cascade Centralized Control Center in River Basin

Abstract. Following the trend of currently intelligent technology development in relevant fields at home and abroad, we planned and designed targets of building intelligent hydroelectric plants and the system's overall architecture. Related ideas were put up with according to the need of real practice.

\section{Introduction}

Water and power companies in different basins in China has successively established automatic systems of cascade centralized control center, achieving optimization,control and management of cascade water and power resources in river basins. Automatic systems have been extensively put into application such as of cascade power plants supervision, hydrological and water dispatching information, generating sets monitoring and long-distance diagnosis,relay protecting, dam safety monitoring and measuring. Technologies are getting mature in building unattended power stations, which makes great contributions to improving the level of secure operation and automatic management of cascade hydroelectric plants as well as laying a solid foundation for intelligence.It is of great significance to propose the idea of building an intelligent automatic system of cascade centralized control center in river basins.

\section{Overall Architecture of Building an Intelligent Cascade Hydroelectric Plant in River Basins}

A. Overall Structure of Automation System of Cascade Hydroelectric Plant in River Basins

According to current operation mode of basin hydroelectric plants,the automatc system adopts a modular design in forms of different layers and zones,formed by organic integrations of a series of intelligent application system with specific functions. To be more specific,layers are divided in line with overall structure of the system while zones with safety level of the system.

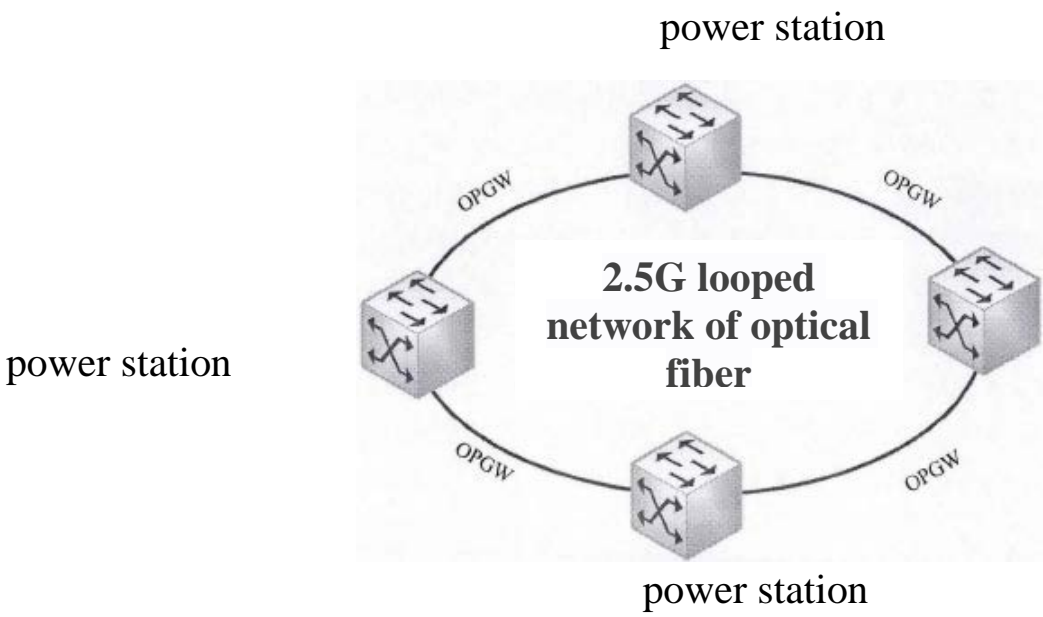

power station 
Figure 1 Topological Structure

\section{B. Communication Network System of Basin Cascade Centralized Control}

Communications network system is not only a basis of application system connecting,data exchanging and information sharing among hydroelectric plants,but also a basic support of building unified information platform for all basin cascade hydroelectric plants. Therefore, it is necessary to make a unified plan and design for computer communication network and configure redundant channels.

1) Principle of maximum power output.With a given water discharge, this principle is mainly used to match the corresponding power generation scheme with maximum power output of the whole hydroelectric plant during the dispatching period.It provides reference for staff to work out long-,middle-,short-term and daily plans.Mathematical model of the principle can be seen as follows:

$$
J=\operatorname{Max} \sum_{i=1}^{T} \sum_{i=1}^{N} E\left(\bar{H}_{i}^{t}, Q_{i}^{t}\right)
$$

(Formula 1)

$E\left(\bar{H}_{i}^{t}, Q_{i}^{t}\right)$ represents power output of Station i during the Period i.The model runs in accordance with the station's economic condition.

At the beginning of the the scheduling period, quantity of water storage in reservoirs at all levels is known as $\mathrm{V}_{1}{ }^{0} \quad \mathrm{i}=\mathrm{l}, \ldots, \mathrm{N}$

At the end of the the scheduling period, quantity of water storage in reservoirs at all levels is known as $\mathrm{V}_{1}^{\mathrm{T}}, \mathrm{i}=1, \ldots, \mathrm{N}$

2) Principle of maximum energy storage

This principle is used by cascade centralized control center to optimize the distribution of total electric load to different hydroelectric plants and generating sets after the superior dispatching department gives out the order,thus maximizing energy storage of the whole cascade hydroelectric plant. Mathematical model of the principle can be seen as follows:

$$
J=\operatorname{Max} \sum_{i=1}^{T} \sum_{j=1}^{N} S\left(\bar{H}_{i}^{\prime}, Q_{i}^{\prime}\right)
$$

(Formula 2)

$S\left(\bar{H}_{i}^{t}, Q_{i}^{\prime}\right)$

represents energy storage of Station i during the Period i.The calculating method is:

$$
\left\{\begin{array}{lc}
\left(q_{i}^{i}-Q_{i}^{t}-y_{i}^{t}\right)\left[\sum_{j=1}^{N} \overline{H_{j}^{\prime}}\right. & i=1 ; t=0, \cdots, T \\
\left(q_{i}^{i}+Q_{i-1}^{t-\tau_{i-1}}+y_{i-1}^{t-\tau_{i-1}}-Q_{i}^{t}-y_{i}^{t}\right) \square \sum_{j=i}^{N} \overline{H_{j}^{t}} & i=2, \cdots, N ; t=0, \cdots, T
\end{array}\right.
$$

(Formula 3)

Constraint on balanced output:

$$
\sum_{i=1}^{N} P\left(\bar{H}_{i}^{t}, Q_{i}^{t}\right)=P_{s}^{t}
$$

(Formula 4)

$P\left(\bar{H}_{i}^{t}, Q_{i}^{t}\right)$ represents optimum total output of active power of Station i during the Period i.The model runs in accordance with the station's economic condition. At the beginning of the dispatching period, quantity of water storage in reservoirs at all levels is known as $\mathrm{V}_{1}{ }^{0}$ $\mathrm{i}=\mathrm{l}, \ldots . ., \mathrm{N}$ 
3) Principle of minimum level over water line limit

This principle is mainly used in the condition that water line control is laid on upstream reservoirs of cascade hydroelectric plants and the water line is out of limitation.Mathematical model of the principle can be seen as follows:

$$
J=\operatorname{Min}\left\{\operatorname{Max}\left\{\delta_{1}, \cdots, \delta_{N}\right\}\right\}
$$

The constraint condition is the same as mathematical model of maximum energy storage.In

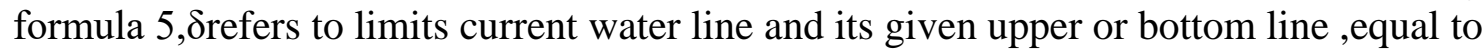

$$
\delta=\left\{\begin{array}{lc}
0 & \left(\underline{Z_{i}} \leq Z_{i} \leq \bar{Z}_{i}\right) \\
Z_{i}-\bar{Z}_{i} & \left(Z_{i}>\bar{Z}_{i}\right) \\
\underline{Z_{i}}-Z_{i} & \left(Z_{i}<\underline{Z_{i}}\right)
\end{array}\right.
$$

(Formula 6)

4) General constraint condition of different mathematical model

Constraint on balanced water yield:

$$
\begin{cases}V_{1}^{t}=V_{1}^{t-1}+\left(q_{1}^{t}-Q_{1}^{t}-y_{1}^{t}\right) \Delta t & i=1 ; t=0, \cdots, T \\ V_{i}^{t}=V_{i}^{i-1}+\left(q_{i}^{t}+Q_{i-1}^{i-t_{j-1}}+y_{i-1}^{i-t_{i-1}}-Q_{i}^{t}-y_{i}^{t}\right) \Delta t & i=2, \cdots, N ; t=0, \cdots, T\end{cases}
$$

(Formula 7)

Constraint on output of the power station:

$$
P_{i}^{t} \leq P_{i}^{t} \leq \overline{P_{i}^{t}} \quad \mathrm{i}=1, \cdots, \mathrm{N}
$$

(Formula 8)

Constraint on water storage of the reservoir:

$$
\underline{V_{i}^{t}} \leq V_{i}^{t} \leq \overline{V_{i}^{t}} \quad \mathrm{i}=1, \cdots, \mathrm{N}
$$

(Formula 9)

Constraint on power output of the power station:

$$
\underline{Q_{i}^{t}} \leq Q_{i}^{t} \leq \overline{Q_{i}^{\prime}} \quad \mathrm{i}=1, \cdots, \mathrm{N}
$$

Communication mode of network of electric optical fiber: core optical network applies MSTP looped network structure based on SDH. Communication mode of telecommunication optical fiber channel: point-to-point fiber transmission channel of 1 or $\mathrm{N} \times 2 \mathrm{Mb} / \mathrm{s}$ formed through single or multi E1(G.703) interface. Emergency satellite communication channel is $64 \mathrm{~kb} / \mathrm{s} \sim 2 \mathrm{Mb} / \mathrm{s}$.Priority of electric optical transmission,the main channel,is high than telecommunication fiber transmission,the back-up channel.

\section{Overall Structure of Intelligent Automatic System of Basin Cascade Hydroelectric Plant.}

Intelligent basin cascade hydroelectric plant is embodied in the construction of a series of highly intelligent automatic systems,such as controls of production process,operation and decision-making and production aided management. These systems, with their own specific functions and clear borders, are relatively separate. They realize a seamless connection by completing their own task and unifying the data model. General principle of AGC software adjustment can be explained as follows. The remaining loan becomes part of equal distributions among joint control generating sets,equal to total active power of the plant minus actual active power that is excluded from AGC adjustment generators. The formula is. 


$$
\left\{\begin{array}{l}
P_{A G C}=P-P_{A G C} \\
P_{i}=P_{A G C} / n
\end{array}\right.
$$

(Formula 11)

$\mathrm{P}_{\mathrm{AGC}}$ represents fixed total active power of AGC adjustment generating sets.P represents the plant's active power dispatched by the provincial power corporation. $\mathrm{P}_{\text {AGC }}$ is equal to the sum of actual power generation that is excluded from the generators.n represents the amount of the generating sets. $\mathrm{P}_{\mathrm{i}}$ represents how much power the Generator i was assigned to generate.

\section{Hierarchical Structure of Intelligent Automatic System of Basin Cascade Hydroelectric Plant}

To build an intelligent basin cascade hydroelectric plant involves production, operation, maintenance, management and so on. This large-scale project with complex structure has a lot to research, a high level of technological difficulties and a long period of construction. Therefore,there are a number of key technical problems remaining to be researched and solved.After curve fitting the discrete points with the function of least squares method coming with origins. 0 software, the fitting curve was drawn as shown in figure 2.

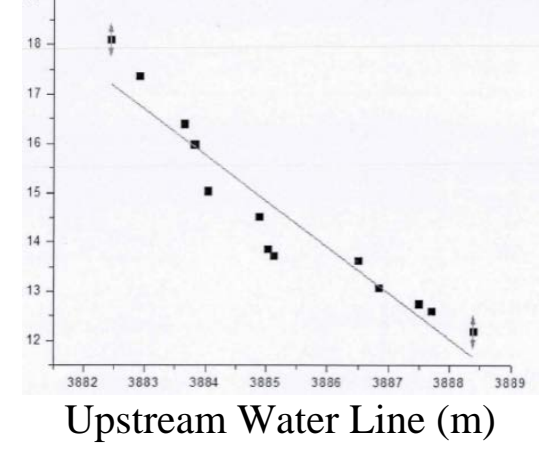

Figure 2 Fitting curve of rate of water consumption and upstream water line

Infrastructures are hardware and software equipment that provide practical environment for the whole process of data collection, transmission, processing storage and presentation. They are also the basis of building an intelligent hydroelectric plant.

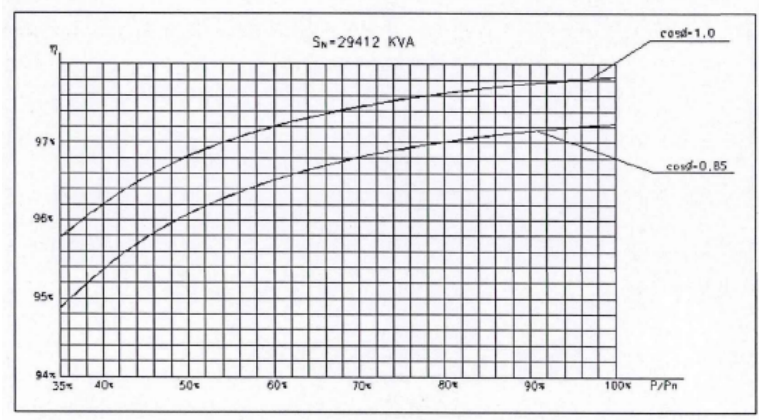

Figure 3 Efficiency curve

Application system is sub-systems of every business which is directly related to the operation, maintenance, and management of the power station. It mainly includes key aspects in intelligence construction such as automation system of basin cascade centralized control center, computer monitoring system of each basin power stations, automation system of hydrological information and water diversion, system of online monitoring and condition based maintenance, safety monitoring system of the dam, protection and measuring system. 


\section{Aspects concerning Construction}

\section{A. Building exclusive data network for basin cascade centralized control center of power stations}

For the automation system of centralized control center to function, it provides a foundation and guarantee to build an exclusive data network for basin cascade centralized control center of power stations and to achieve reliable communication between centralized control center and power stations at all levels. We achieve centralized control over power stations at all levels, cooperative optimizing dispatching. Connections with the centralized center are flexibly made through corresponding network communication.

\section{B. Building Unified Information Platform for Basin Cascade Power Stations}

After building basic supporting system such as computer communication network system,practical operation environment for unified information platform,equipment of data exchange and central storage is built among basin cascade power stations. Intelligence construction of different application systems is based on real process through unified transmission protocol and network interface, establishing information center of all basin cascade power stations. Therefore, this automation system will gain higher level of operation and management and better comprehensive economic results.

\section{Building Automatic System for Power Dispatching among Basin Cascade Centralized Control Center of Power Stations}

To build automation system for power dispatching among basin cascade centralized control center of power stations, we adopt layered and distributed architecture:upper dispatching level, cascade centralized control center level and station level. We set up a automation system for power dispatching in the second layer for long-distance monitoring and control over all basin cascade power stations and the conditions of facilities like spillway sluices.

\section{Building Automatic System for Water Dispatching among Basin Cascade Centralized Control Center of Power Stations}

To build automation system for water dispatching among basin cascade centralized control center of power stations,we adopt distributed architecture:main water dispatching system,flood prevention system, hydrological interface subsystemand so forth. All functions of the system are distributed in nodes of the network,as they seek to satisfy the need of operation staff,to release their working intensity, and to achieve cascade economic targets of intelligent monitoring, predicting, dispatching and managing.

\section{E. Planning of Building Intelligent Automatic System of Basin Cascade Power Stations}

Planning of building intelligent automation system of basin cascade power stations mainly includes building information model for intelligent hydroelectric plant in line with IEC 61850,building a new generation of platform of auto-control system through advanced technology such as sensors, electronic, information, control and intelligent analysis.

\section{Summary}

We build intelligent automatic system of basin cascade centralized control center while intelligent basin power plant is under construction. Through unified data integration platform, we achieve joint dispatching among reservoirs, long-distance centralized control over basin cascade power stations and optimized operation of generating sets. It is of great significance to improve the 
level of intelligent decision-making in the operation and management of basin cascade power stations, release working intensity and improve economic performance.

\section{References}

[1]Wang Gang,Pang Zhengzheng,Hou Fei,Gao Zhiyong,Yang Yonghong:Design of computer monitoring system of cascade power station centralized control center in Ya-lung basin [J].Yunnan Water Power,2014,06:115-118.

[2]Xie Zhenyu:Research on centralized monitoring and joint dispatching system of Cascade Hydro-power Station Groups [J].Technology \& Business,2015,02:87-91.

[3]Wang Xu,Yang Weili,Cai Heng:Overview of Research on Cascade Power Station Centralized Control Center in the middle reaches of Jinsha River [J].Hydrogen-power Generation,2015,05:40-43.

[4]Liu Shuyu,ZhangWeili,Wu Yuan:Brief Analysis on the Planning of Cascade Centralized Control and Intelligent hydro-power Stations in Yuan Area [J].Mechanical \& Electrical Technique of Hydropower Station,2015,07:14-17+45+84.

[5]Wang Gang,Dou Haini:Design and Application of centralized contr sysyem over cascade hydro-power station Groups [J].Yangtze River,2015,11:93-95+103. 